PROCEEDINGS OF THE

AMERICAN MATHEMATICAL SOCIETY

Volume 126, Number 11, November 1998, Pages 3299-3305

S 0002-9939(98)04624-3

\title{
PROJECTIVE MODULES AND HILBERT SPACES WITH A NEVANLINNA-PICK KERNEL
}

\author{
ROBERT S. CLANCY AND SCOTT MCCULLOUGH
}

(Communicated by Palle E. T. Jorgensen)

\begin{abstract}
In this paper we solve a mapping problem for a particular class of Hilbert modules over an algebra multipliers of a diagonal Nevanlinna-Pick (NP) kernel. In this case, the regular representation provides a multiplier norm which induces the topology on the algebra. In particular, we show that, in an appropriate category, a certain class of Hilbert modules are projective. In addition, we establish a commutant lifting theorem for diagonal NP kernels.
\end{abstract}

\section{INTRODUCTION}

Douglas and Paulsen [DP89] define a Hilbert module as a Hilbert space $\mathcal{H}$ together with a continuous action of a function algebra $\mathbb{A}$. The notion of a Hilbert module allows some interesting questions in operator theory to be expressed via certain natural algebraic constructions. We consider below both a reproducing kernel Hilbert space $H^{2}(k)$, where $k$ is an NP kernel, which for expository purposes we assume to be diagonal, and the Hilbert modules over the algebra of bounded multipliers of $H^{2}(k)$. The regular representation allows the identification of an element, $f \in H^{2}(k)$, with a (possibly unbounded) multiplication operator, $M_{f}$, with symbol $f$. The algebra of bounded multipliers then consists of those multipliers with finite operator norm.

The full power of the commutant lifting theorem [FF90] is that given contractions $T$, and $T^{\prime}$ acting on Hilbert spaces $\mathcal{H}$, and $\mathcal{H}^{\prime}$, respectively, and a contractive intertwining $A: \mathcal{H} \rightarrow \mathcal{H}^{\prime}$, there exists a contractive intertwining of the minimal isometric dilations of $T$ and $T^{\prime}$. In the sequel, we obtain a commutant lifting theorem when $\mathcal{H}$ and $\mathcal{H}^{\prime}$ are Hilbert modules over the algebra of multipliers of $H^{2}(k)$. This lifting theorem will follow from the solution of a mapping problem which naturally arises in homological constructions. Indeed, it is the solution of this mapping problem which shows certain Hilbert modules to be projective, in an appropriate category.

\section{Results}

The operator of multiplication by $z$ on a reproducing kernel Hilbert space, where the kernel $k$ is a Nevanlinna-Pick kernel (NP for short), enjoys many of the properties of the unilateral shift operator [Agl90a]. In this paper we establish a commutant

Received by the editors January 17, 1997 and, in revised form, March 28, 1997.

1991 Mathematics Subject Classification. Primary 47A20; Secondary 46E22.

(C)1998 American Mathematical Society 
lifting theorem for diagonal NP kernels. Explicitly, our kernel has the form

$$
k(z, \zeta)=\sum_{0}^{\infty} a_{n} z^{n} \bar{\zeta}^{n}
$$

where $a_{0}=1$, and $a_{n}>0$. We also assume $k$ has a positive radius of convergence about $(0,0)$ and

$$
\frac{a_{j}}{a_{j+1}} \leq C^{2}
$$

When $a_{n}=1$ for each $n, k$ is the Szego kernel; when $a_{n}=n+1, k$ is the Bergman kernel; and when $a_{n}=\frac{1}{n+1}, k$ is the Dirichlet kernel.

Since $k(0,0)=1$, near $(0,0)$ we have

$$
\frac{1}{k}(z, \zeta)=1-\sum_{n=1}^{\infty} b_{n} z^{n} \bar{\zeta}^{n}
$$

We note for future use that, for $n \geq 1$,

$$
a_{n}=\sum_{s=1}^{n} b_{s} a_{n-s} .
$$

In this context, $k$ is an NP kernel if $b_{n} \geq 0$ for each $n \geq 1$. It is easy to verify that the Szego kernel is an NP kernel and that the Bergman kernel is not. To show that the Dirichlet kernel is an NP kernel requires some effort [SS62] [Agl90b]. Let $H^{2}(k)$ denote the Hilbert space (equals complex separable or finite dimensional Hilbert space) obtained by closing up analytic polynomials in the inner product determined by

$$
\left\langle z^{s}, z^{t}\right\rangle= \begin{cases}\frac{1}{a_{s}}, & \text { if } s=t \\ 0, & \text { if } s \neq t\end{cases}
$$

Let $k_{\ell}(z)=a_{\ell} \zeta^{\ell}$. Since $\left\langle z^{s}, k_{t}\right\rangle=\delta_{s, t}$, and polynomials are dense in $H^{2}(k),\left\{k_{\ell}\right\}$ is a dual basis to $\left\{z^{n}\right\}$.

The condition (2.2) implies the operator $S_{k}$ on $H^{2}(k)$ defined by $S_{k} z^{n}=z^{n+1}$ is bounded with $\left\|S_{k}\right\| \leq C$. Standard computations show that

$$
S_{k}^{*} k_{\ell}=k_{\ell-1},
$$

where we interpret $k_{s}=0$, if $s<0$.

We will state the result in the language of modules. Given a Hilbert space $\mathcal{H}$, let $\mathcal{L}(\mathcal{H})$ denote the bounded operators on $\mathcal{H}$. Each $J \in \mathcal{L}(\mathcal{H})$ determines a module over $\mathbb{C}[z]$ by

$$
p \cdot h=P(J) h,
$$

for $p \in \mathbb{C}[z]$ and $h \in H$. We denote this module $(J, \mathcal{H})$. For our purposes, a module $(T, \mathcal{M})$ is a ${ }^{*}$-submodule of $(J, \mathcal{H})$ if $\mathcal{M}$ is a subspace of $\mathcal{H}$ which is invariant for $J^{*}$ and $T=P_{\mathcal{M}} J$, where $P_{\mathcal{X}}$ is the orthogonal projection of $\mathcal{H}$ onto $\mathcal{M}$. Since, for $p \in \mathbb{C}[z], p(T) P_{\mathcal{M}}=P_{\mathcal{M}} p(J)$,

$$
P_{\mathcal{M}}:(J, \mathcal{H}) \mapsto(T, \mathcal{M})
$$

is a module homomorphism. In what follows $\mathcal{H}^{2}(k)=\bigoplus_{j=0}^{\infty} H^{2}(k)$ and $\mathcal{S}_{k}=$ $\bigoplus_{j=0}^{\infty} S_{k}$ 
Theorem 2.1. Let $k$ be a diagonal NP kernel. If $(C, \mathcal{M})$ is a ${ }^{*}$-submodule of $\left(\mathcal{S}_{k}, \mathcal{H}^{2}(k)\right)$ and if $f:\left(\mathcal{S}_{k}, \mathcal{H}^{2}(k)\right) \mapsto(C, \mathcal{M})$ is a bounded module homomorphism, then there exists a module homomorphism $F:\left(\mathcal{S}_{k}, \mathcal{H}^{2}(k)\right) \mapsto\left(\mathcal{S}_{k}, \mathcal{H}^{2}(k)\right)$ such that $P_{\mathcal{M}} F=f$ and $\|F\|=\|f\|$. Diagrammatically,

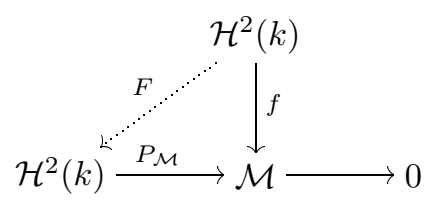

Theorem 2.1 prepares the way for the study of the homological algebra of *submodules of $\left(\mathcal{S}_{k}, \mathcal{H}^{2}(k)\right)$, an investigation pursued by the first author in forthcoming work. Indeed, let $\mathcal{C}$ be the category whose objects are ${ }^{*}$-submodules of $\mathcal{H}^{2}(k)$, and morphisms are bounded module maps. Let $\mathcal{E}$ be the class of all sequences

$$
\cdots \longrightarrow \mathcal{M}^{\prime} \stackrel{\mu^{\prime}}{\longrightarrow} \mathcal{M} \stackrel{\mu}{\longrightarrow} \mathcal{M}^{\prime \prime} \longrightarrow \cdots
$$

in which each morphism is a partial isometry, and for $\mu^{\prime}$ and $\mu$ successive morphisms in the sequence we have image $\left(\mu^{\prime}\right)=\operatorname{kernel}(\mu)$. Decree the elements of $\mathcal{E}$ to be the exact sequences of $\mathcal{C}$, and define an object $\mathcal{P} \in \mathcal{C}$ to be projective if for every diagram with bottom row exact,

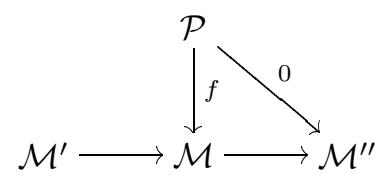

there exists a bounded module map $F: \mathcal{P} \rightarrow \mathcal{M}^{\prime}$ making the diagram commute. In this setting we have the following

Corollary 2.2. Let $\mathcal{C}, \mathcal{E}, \mathcal{H}^{2}(k)$ be as above. $\mathcal{H}^{2}(k)$ is projective in $\mathcal{C}$.

The notion of an NP kernel and a version of Theorem 2.1 suitable for NevanlinnaPick interpolation is due to Agler [Agl90b]. Douglas and Paulsen [DP89], Carlson and Clark [CC95], and Ferguson [Fer96] have produced results for $k$, the Szego kernel. Choosing $k$ to be the Szego kernel in Theorem 2.1 gives commutant lifting [FS70] for contractions $C$ and $C^{\prime}$ whose minimal isometric dilations have no unitary summand as can be seen immediately from the following corollary.

Corollary 2.3. If $\mathcal{M}$ and $\mathcal{M}^{\prime}$ are invariant for $\mathcal{S}_{k}^{*}, X$ is a bounded operator from $\mathcal{M}$ to $\mathcal{M}^{\prime}, C=P_{\mathcal{M}} \mathcal{S}_{k} P_{\mathcal{M}}, C^{\prime}=P_{\mathcal{M}^{\prime}} \mathcal{S}_{k} P_{\mathcal{M}^{\prime}}$, and $X C=C^{\prime} X$, then there exists $Y \in \mathcal{L}\left(\mathcal{H}^{2}(k)\right)$ such that $\left.Y^{*}\right|_{\mathcal{M}}=X^{*},\|Y\|=\|X\|$, and $Y \mathcal{S}_{k}=\mathcal{S}_{k} Y$.

The remainder of the paper is devoted to proofs of Theorem 2.1 and Corollaries 2.2 and 2.3 .

\section{THE PROOFS}

Throughout we assume $k$ is an NP kernel and $a_{j}$ and $b_{j}$ are as in the introduction. For expository purposes, we make use of tensor product notation. Fix a (separable) 
Hilbert space $\mathcal{H}$. We identify $\mathcal{H}^{2}(k)$ with $\mathcal{H} \otimes H^{2}(k)$ and $\mathcal{S}_{k}$ with $I \otimes S_{k}$, where $I$ is the identity on $\mathcal{H}$. In particular, $g \in \mathcal{H}^{2}(k)$ can be represented as

$$
g=\sum g_{n} \otimes z^{n}
$$

for some sequence $g_{n}$ from $\mathcal{H}$, where the series converges in norm; and

$$
\mathcal{S}_{k} g=\sum g_{n} \otimes z^{n+1}
$$

Lemma 3.1. Let $A: \mathcal{H} \mapsto \mathcal{M}$. Suppose $(T, \mathcal{N})$ is a ${ }^{*}$-submodule of $\left(\mathcal{S}_{k}, \mathcal{H}^{2}(k)\right)$ and $(C, \mathcal{M})$ is a ${ }^{*}$-submodule of $(T, \mathcal{N})$. If

$$
\sum_{j=0}^{N} a_{j}\left(C^{j} A\right)\left(C^{j} A\right)^{*} \leq I
$$

for all $N$, and if $T^{j} A=T C^{j-1} A$ for all $j \geq 1$, then

$$
\sum_{j=1}^{M} a_{j}\left(T^{j} A\right)\left(T^{j} A\right)^{*} \leq I,
$$

for all $M$.

Proof. For a fixed $M$ we have, using (2.6) repeatedly,

$$
\left\langle\left(\sum_{j=1}^{M} b_{j} S_{k}^{j} S_{k}^{* j}\right) k_{s}, k_{t}\right\rangle=\sum_{j=1}^{M} b_{j}\left\langle k_{s-j}, k_{t-j}\right\rangle .
$$

If $s \neq t$ then the sum is 0 . In the case $s=t$, (3.3) equals $\sum_{j=1}^{M} b_{j} a_{s-j}$ which is less than $\sum_{j=1}^{s} b_{j} a_{s-j}=a_{s}$, where we interpret $a_{\ell}=0$ for $\ell<0$. It follows that

$$
\sum_{j=1}^{M} b_{j} S_{k}^{j} S_{k}^{* j} \leq I
$$

for each $M$. It is immediate that (3.4) holds with $S_{k}$ replaced by $\mathcal{S}_{k}$. With $T=$ $P_{\mathcal{N}} \mathcal{S}_{k}$ we have

$$
T^{j} T^{* j}=P_{\mathcal{N}} \mathcal{S}_{k}^{j} \mathcal{S}_{k}^{* j} P_{\mathcal{N}}
$$

Since $b_{j} \geq 0$, it now follows from (3.5) that (3.4) holds with $T$ in place of $S_{k}$. 
We can now estimate,

$$
\begin{aligned}
I & \geq \sum_{s=1}^{M} b_{s} T^{s} T^{* s} \\
& \geq \sum_{s=1}^{M} b_{s} T^{s}\left(\sum_{j=0}^{M-s} a_{j} C^{j} A A^{*} C^{* j}\right) T^{* s} \\
& =\sum_{s=1}^{M} \sum_{j=0}^{M-s} b_{s} a_{j} T^{j+s} A A^{*} T^{*(j+s)} \\
& =\sum_{n=1}^{M} \sum_{s=1}^{n} b_{s} a_{n-s} T^{n} A A^{*} T^{* n} \\
& =\sum_{n=1}^{M} a_{n} T^{n} A A^{*} T^{* n} .
\end{aligned}
$$

The strategy is to use Lemma 3.1 and the Parrott Lemma [Par70] to do one step extensions. Accordingly, let $q$ denote the least natural number such that $\mathcal{H} \otimes\left[k_{q}\right]=\left\{h \otimes k_{q}: h \in \mathcal{H}\right\}$ is not a subspace of $\mathcal{M}$. Let $\mathcal{N}$ denote the closure of the span of the subspaces $\mathcal{M}$ and $\mathcal{H} \otimes\left[k_{q}\right]$. Since $\mathcal{S}_{k}^{*}$ maps $\mathcal{H} \otimes\left[k_{q}\right]$ into $\mathcal{H} \otimes\left[k_{q-1}\right] \subset \mathcal{M}$, $\mathcal{N}$ is invariant for $\mathcal{S}_{k}^{*}$. Thus, with $T=P_{\mathcal{N}} \mathcal{S}_{k},(T, \mathcal{N})$ is a ${ }^{*}$-submodule of $\left(\mathcal{S}_{k}, \mathcal{H}^{2}(k)\right)$ and $(C, \mathcal{M})$ is a ${ }^{*}$-submodule of $(T, \mathcal{N})$. Also observe that, as the range of $T^{*}$ is in $\mathcal{M}$

$$
T(\mathcal{N} \ominus \mathcal{M})=\{0\} .
$$

Lemma 3.2. There exists a module homomorphism $F:\left(\mathcal{S}_{k}, \mathcal{H}^{2}(k)\right) \mapsto(T, \mathcal{N})$ such that $P_{\mathcal{M}} F=f$, and $\|F\| \leq\|f\|$.

Proof. Without loss of generality, we may assume that $\|f\|=1$. Given a Hilbert space $\mathcal{K}$ and a bounded operator $G: \mathcal{H}^{2}(k) \mapsto \mathcal{K}$, define $G_{j}: \mathcal{H} \mapsto \mathcal{K}$ by $G_{j} h=$ $G\left(h \otimes z^{j}\right)$. The matrix of $G^{*}$ with respect to the orthogonal decomposition of $\mathcal{H}^{2}(k)=\bigoplus\left(\mathcal{H} \otimes\left[z^{j}\right]\right)$ is then

$$
G=\left(\begin{array}{llll}
G_{0}^{*} & a_{1} G_{1}^{*} & a_{2} G_{2}^{*} & \ldots
\end{array}\right)^{t} .
$$

Thus $\|G\| \leq I$ if and only if

$$
I \geq G G^{*}=\sum a_{j} G_{j} G_{j}^{*} .
$$

Since $f \mathcal{S}_{k}^{j}=C^{j} f$, we have $f_{j}=C^{j} f_{0}$. Hence from (3.8),

$$
I \geq \sum a_{j}\left(C^{j} f_{0}\right)\left(C^{j} f_{0}\right)^{*} .
$$

To obtain $P_{\mathcal{M}} F=f$, with respect to the orthogonal decomposition

$$
\mathcal{N}=(\mathcal{N} \ominus \mathcal{M}) \oplus \mathcal{M},
$$

our desired $F$ must have the form

$$
F_{j}=\left(\begin{array}{l}
g_{j} \\
f_{j}
\end{array}\right)
$$


for some $g: \mathcal{H}^{2}(k) \mapsto \mathcal{N} \ominus \mathcal{M}$. The condition that $F$ is a module homomorphism is equivalent to $F \mathcal{S}_{k}^{n}=T^{n} F$, for $n \geq 1$. Consequently, given $F_{0}, F$ is a module homomorphism if and only if

$$
F_{j}=T^{j} F_{0}
$$

(assuming $F$ is a bounded operator). Notice that (3.6) implies $F_{j}=T^{j} f_{0}$, for $j \geq 1$. In particular, for $j \geq 1, g_{j}$ is already determined. The only choice is of $g_{0}$.

The matrix of $F$ with respect to the orthogonal decomposition of $\mathcal{H}^{2}(k)$ as $(\mathcal{H} \otimes[1]) \oplus(\mathcal{H} \otimes[1])^{\perp}$ and $\mathcal{N}$ as $(\mathcal{N} \ominus \mathcal{M}) \oplus \mathcal{M}$ has the form

$$
F=\left(\begin{array}{cc}
g_{0} & a \\
b & c
\end{array}\right)
$$

where $g_{0}$ is to be chosen,

$$
\left(\begin{array}{ll}
b & c
\end{array}\right)=f
$$

and

$$
\left(\begin{array}{l}
a \\
c
\end{array}\right)=\left(\begin{array}{lll}
\sqrt{a_{1}} F_{1} & \sqrt{a_{2}} F_{2} & \ldots
\end{array}\right) .
$$

We will show that the operator in (3.12) has norm at most one. Since $\|f\| \leq 1$, from the Parrott Lemma there exists $g_{0}$ such that $\|F\| \leq 1$. This will then complete the proof of Theorem 2.1. Now the operator of (3.12) has norm at most one if and only if

$$
I \geq \sum_{j=1}^{M} a_{j} F_{j} F_{j}^{*}
$$

holds for all $M$. Using $F_{j}=T^{j} f_{0}$ and $T^{j} f_{0}=T C^{j-1} f_{0}$ for $j \geq 1$, and (3.9), we see (3.13) holds by Lemma 3.1.

Theorem 2.1 is now established by repeatedly applying Lemma 3.2. We omit the details. Let

$$
\mathcal{N}^{\prime} \underset{P_{\mathcal{N}}}{\longrightarrow} \mathcal{N} \longrightarrow \mathcal{N}^{\prime \prime}
$$

be a member of the class $\mathcal{E}$ of exact sequences. To see that $\mathcal{H}^{2}(k)$ is projective as described above we consider the following

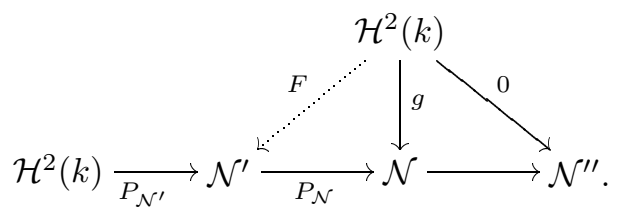

Hence Theorem 2.1 now implies we can complete the diagram with a map $F$ : $\mathcal{H}^{2}(k) \rightarrow \mathcal{H}^{2}(k)$, by letting $\mathcal{M}=\mathcal{N}, g=f$, and $\mathcal{N}^{\prime \prime}=0$. Corollary 2.2 follows since $\hat{F}:=P_{\mathcal{N}^{\prime}} \circ F: \mathcal{H}^{2}(k) \rightarrow \mathcal{N}^{\prime}$ solves the following diagram

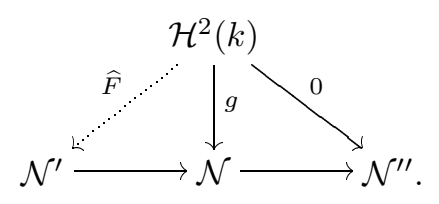


From the usual diagram

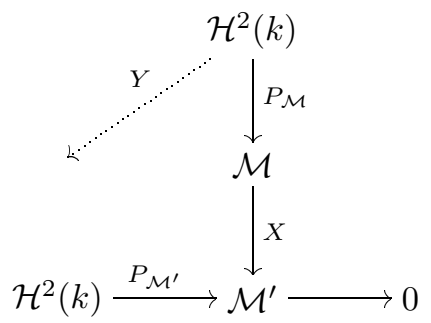

it is seen that Corollary 2.3 is an immediate consequence of Theorem 2.1.

\section{REFERENCES}

[Ag190a] J. Agler, A disconjugacy theorem for toeplitz operators, American Journal of Mathematics 112 (1990), 1-14. MR 91b:47048

[Agl90b] J. Agler, Interpolation, Journal of Functional Analysis, To appear.

[CC95] Carlson,J.F. and Clark,D.N., Cohomology and extensions of hilbert modules, Journal of Functional Analysis 128 (1995), 278-306. MR 96a:46129

[DP89] Douglas, Ronald G. and Paulsen, Vern I., , Pitman research notes in mathematics series, no. 217, Harlow, Essex, England : Longman Scientific and Technical; New York: Wiley, 1989. MR 91g:46084

[Fer96] Sarah H. Ferguson, Polynomially bounded operators and ext groups, Proc. Amer. Math. Soc. 124 (1996), no. 9, 2779-2785. MR 96k:47012

[FF90] Foias, Ciprian and Frazho, Arthur E., The commutant lifting approach to interpolation problems, Operator Theory: Advances and Applications, vol. 44, Birkhauser Verlag, 1990. MR 92k:47033

[FS70] Foias, C.and Sz-Nagy, B., Harmonic analysis of operators an hilbert spaces, North Holland, 1970. MR 43:947

[Par70] S.K. Parrot, Unitary dilations for commuting contractions, Pacific Journal of Math. (1970), 481-490. MR 42:3607

[SS62] Shapiro,H.S. and Shields,A.L., On the zeroes of functions with finite dirichlet integral and some related function spaces, Math. Z. 80 (1962), 217-229. MR 26:2617

Department of Mathematics, 358 Little Hall, University of Florida, Gainesville, FLORIDA 32611

E-mail address: rsc@math.ufl.edu

E-mail address: sam@math.ufl.edu 